\title{
Isolation and Characterization of APETALA3 Orthologs and Promoters from the Distylous Fagopyrum esculentum
}

\author{
Lingtian Zeng, Jiao Zhang, Xuan Wang and Zhixiong Liu *
}

check for updates

Citation: Zeng, L.; Zhang, J.; Wang,

X.; Liu, Z. Isolation and

Characterization of APETALA3

Orthologs and Promoters from the

Distylous Fagopyrum esculentum.

Plants 2021, 10, 1644. https://

doi.org/10.3390/plants10081644

Academic Editor: Agnes Farkas

Received: 20 July 2021

Accepted: 9 August 2021

Published: 10 August 2021

Publisher's Note: MDPI stays neutral with regard to jurisdictional claims in published maps and institutional affiliations.

Copyright: (c) 2021 by the authors. Licensee MDPI, Basel, Switzerland. This article is an open access article distributed under the terms and conditions of the Creative Commons Attribution (CC BY) license (https:/ / creativecommons.org/licenses/by/ $4.0 /)$.
College of Horticulture and Gardening, Yangtze University, Jingzhou 434025, China; 202072800@yangtzeu.edu.cn (L.Z.); 201871411@yangtzeu.edu.cn (J.Z.); 201872469@yangtzeu.edu.cn (X.W.)

* Correspondence: zxliu@yangtzeu.edu.cn; Tel.: +86-716-8066260

\begin{abstract}
Common buckwheat (Fagopyrum esculentum) produces distylous flowers with undifferentiated petaloid tepals, which makes it obviously different from flowers of model species. In model species Arabidopsis, APETALA3 (AP3) is expressed in petal and stamen and specifies petal and stamen identities during flower development. Combining with our previous studies, we found that smallscale gene duplication (GD) event and alternative splicing (AS) of common buckwheat AP3 orthologs resulted in FaesAP3_1, FaesAP3_2 and FaesAP3_2a.FaesAP3_2 and FaesAP3_2a were mainly expressed in the stamen of thrum and pin flower. Promoters functional analysis suggested that intense GUS staining was observed in the whole stamen in pFaesAP3_2::GUS transgenic Arabidopsis, while intense GUS staining was observed only in the filament of stamen in pFaesAP3_1::GUS transgenic Arabidopsis. These suggested that FaesAP3_1 and FaesAP3_2 had overlapping functions in specifying stamen filament identity and work together to determine normal stamen development. Additionally, FaesAP3_2 and FaesAP3_2a owned the similar ability to rescue stamen development of Arabidopsis ap3-3 mutant, although AS resulted in a frameshift mutation and consequent omission of the complete PI-derived motif and euAP3 motif of FaesAP3_2a. These suggested that the MIK region of AP3-like proteins was crucial for determining stamen identity, while the function of AP3-like proteins in specifying petal identity was gradually obtained after AP3 Orthologs acquiring a novel C-terminal euAP3 motif during the evolution of core eudicots. Our results also provide a clue to understanding the early evolution of the functional specificity of euAP3-type proteins involving in floral organ development in core eudicots, and also suggested that FaesAP3_2 holds the potential application for biotechnical engineering to develop a sterile male line of F. esculentum.
\end{abstract}

Keywords: APETALA3-like gene; Fagopyrum esculentum; MADS-box gene; promoter; stamen development

\section{Introduction}

Common buckwheat (Fagopyrum esculentum) is a gluten-free pseudo-cereal crop mainly cultivated in Europe, North America and Asian for human consumption and forage [1,2]. Buckwheat grains are rich in bioactive compounds, such as rutin, quercetin, polysaccharides and dietary fiber, etc., but with low-calories. Hence, common buckwheat is increasingly demanded and recognized for its highly effective as a functional food with health benefits and illness prevention throughout recent years [1]. However, common buckwheat is an obligate outcrossing crop with heteromorphic self-incompatibility due to its distylous flowers, with population being equally composed of plants with thrum flowers (short pistil combined with long stamen and large pollen grains) and plants with pin flowers (long pistil combined with short stamen and small pollen grains) (Figure 1), and legitimate cross-pollinations occur strictly between anthers and stigmas of equivalent height in different floral morphs, which make it hard for hybrid breeding and result in low yield $[3,4]$. Improving the yield stability and efficiency of breeding programs requires a better understanding the developmental genetics of distylous flowers in common buckwheat. 
Exploring the underlying molecular mechanism of heteromorphic self-incompatibility of common buckwheat has continued for a quarter of a century and has made considerable progress in understanding of the molecular basis of heteromorphic SI in recent years [4-6]. However, the molecular mechanisms responsible for the form and development of the dimorph flower remain to be elucidated in common buckwheat. Furthermore, F. esculentum belongs to family Polygonaceae in the order Caryophyllales, an early diverging core eudicots clade, and produces distylous flowers with undifferentiated petaloid tepals, representing a considerable difference with most core eudicots and major crops flowers, which make it an excellent model for exploring floral organ specification and evolution [7-9].

In Arabidopsis thaliana, the B-class MADS-box genes APETALA3 (AP3) and PISTILLATA $(P I)$ work together to control the formation of petals and stamens during Arabidopsis flower development [10]. The AP3-like and PI-like genes originated from a duplication event of ancient class $\mathrm{B}$ genes that preceded the origin of extant angiosperms [11]. In addition, another major gene duplication event in the AP3/DEFICIENS (DEF) lineage occurred close to, or at the base of, the core eudicot origination, and resulted in the euAP3-like and TM6-like genes [12]. Moreover, most euAP3-like genes express in petals and stamens and specify petal and stamen development, while most TM6-like genes show a broader expression zone in flowers but mainly regulate stamen development [13,14]. Moreover, more and more studies suggested that small-scale gene duplication could also lead to subor neo-functionalization of AP3-like genes in different taxa [14-16].

Here, we found that a small-scale gene duplication (GD) event of buckwheat AP3 ortholog resulted in FaesAP3_1 and FaesAP3_2. Furthermore, alternative splicing (AS) generates two transcript isoforms from the FaesAP3_2 after the gene duplication. Both AS isoforms, FaesAP3_2 and FaesAP3_2a, differ by 32 nucleotides in length and result from alternative 5 'splice-site selection. Our previous study suggested that FaesAP3_1 is involved only in stamen development in F. esculentum [9]. In the present study, the functional diversification of buckwheat $A P 3$-like gene was analyzed by characterizing their promoters. In addition, the function of FaesAP3_2 and FaesAP3_2a specifying floral organ identity were analyzed by assessing their ability to rescue the phenotype of Arabidopsis ap3-3 mutant. The possible impacts of GD and AS on the function of three buckwheat AP3-like genes were investigated, and the functional diversification among them was proposed. Our findings also provide clues for tracing the structure and functional evolution of euAP3-type genes in the early diverging core eudicots.
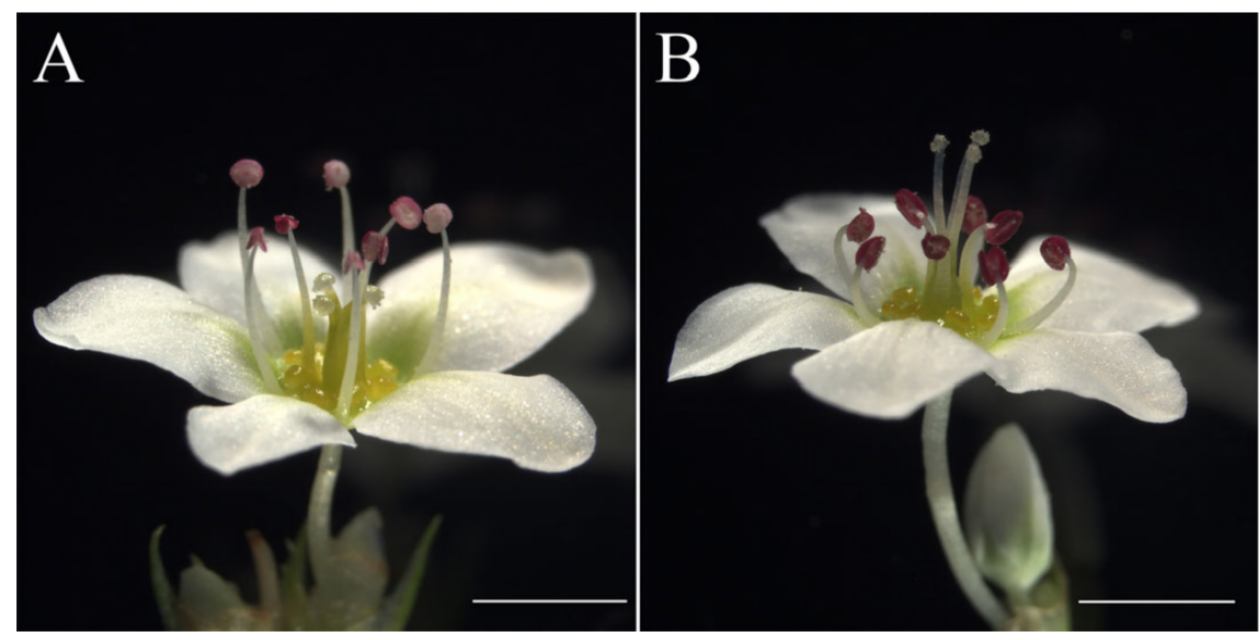

Figure 1. Distylous flower of F. esculentum. (A) Thrum flower with short pistil and long stamen; (B) pin flower with long pistil and short stamen. Scale bar $=2 \mathrm{~mm}$. 


\section{Results}

2.1. Isolation and Characterization of FaesAP3_2 and FaesAP3_2a from F. esculentum

The 891 bp FaesAP3_2 cDNA contains a 732 bp ORF (Open Reading Frame, ORF) encoding 243 amino acids (aa) (Genbank accession number: MN016949.1), while the ORF of the FaesAP3_2a is only 615 bp and encodes 204 aa (Genbank accession number: MN016950.1). Moreover, genomic DNA sequence of FaesAP3_2 (Genbank accession number: MN016951.1) is 2214 bp consisting of seven exons and six introns. Sequences alignment revealed that FaesAP3_2 and FaesAP3_2a were derived from the consensus pre-mRNA, and both variants differed at the exon 6-intron 6 splice junction sites which resulted in a $32 \mathrm{bp}$ nucleotides addition in the sixth exon of Faes AP3_2a from alternative 5 'splice-site selection than the sixth exon of the FaesAP3_2 (Figure 2) $[17,18]$. Phylogenetic tree analysis grouped FaesAP3_2 into euAP3 lineage (Figure 3), and the protein sequence also showed $67.89 \%$ identity with the FaesAP3_1 (Genbank accession number: AFO83616.1), another common buckwheat AP3-like MADS-box transcription factor. The gene was designated as FaesAP3_2 (Fagopyrum esculentum APETALA3_2). Proteins alignment shows that FaesAP3_2 protein comprises a 27 amino acids (aa) seldom seen $\mathrm{N}$-terminal extension region, a 57 aa highly conserved MADS-box domain (28-84), a 82 aa moderately conserved K domain (114-195) in the middle region and a 48 aa variable C-terminal region (196-243), but with two conserved motifs: PI-derived motif and an euAP3 motif (Figure 3) [12,19]. Moreover, FaesAP3_2 includes three putative amphipathic $\alpha$-helices designated as K1 (114-135), K2 (148-162) and K3 (170-205) subdomains containing conserved hydrophobic amino acids residues at the $\mathrm{a}$ and $\mathrm{d}$ positions in the (abcdefg)n heptad repeats [19]. The $32 \mathrm{bp}$ nucleotides addition in the sixth exon of FaesAP3_2a results in frameshift mutation and consequent omission of the complete PI-derived motif and euAP3 motif (Figure 4), two key conserved motifs located at the C-terminal region of the euAP3-like transcription factors that are involved in mediating protein-protein interactions [12].

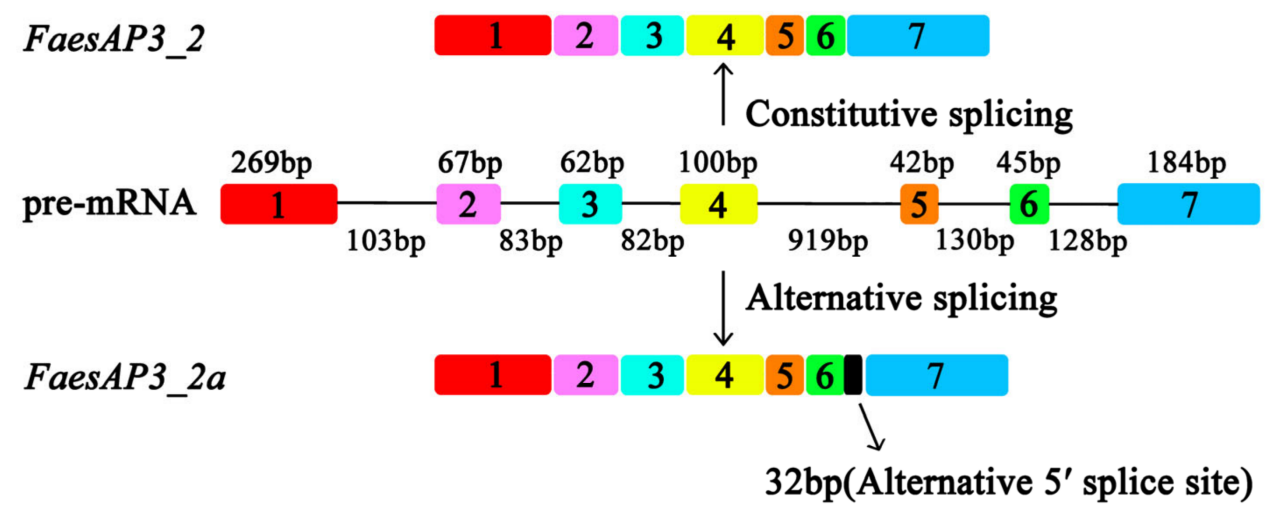

Figure 2. Alternative splicing of FaesAP3_2. Color boxes indicate exons while black lines indicate introns of FaesAP3_2 gene. 


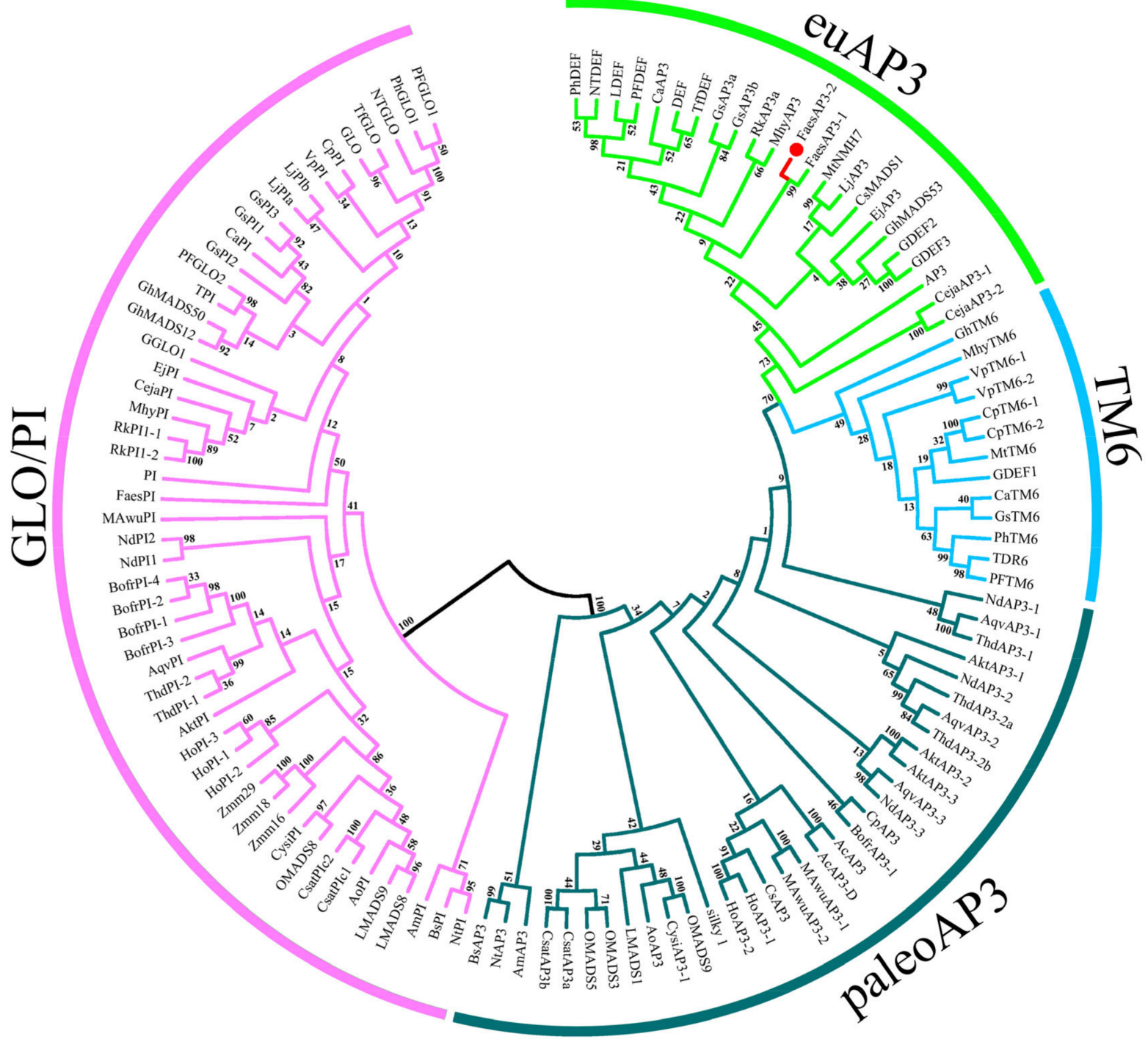

Figure 3. Phylogenetic tree of FaesAP3_2 and other B-class MADS-box transcription factors from different clades of angiosperms.

\subsection{Expression Analysis of FaesAP3_2 and FaesAP3_2a}

FaesAP3_2 was mainly expressed in stamen of thrum and pin flower, but FaesAP3_2a transcript was detected only in stamen (Figure 5A,B). Moreover, weak transcripts of FaesAP3_2 was also observed in tepal, gynoecia and 4-day-old fruit of pin and thrum flowers (Figure 5A). FaesAP3_2 and FaesAP3_2a expressions became apparent after stamen primodium emerge in thrum and pin flower buds (Figure 6A-C). In addition, FaesAP3_2 expression increased constantly and reached the peak until mononuclear microspore at Periphery formation and tepal enclosing in thrum flower buds (T4), but microspore mother cells meiosis and microspores tetrads formation occurred in the pin flower bud (P3), then FaesAP3_2 expression began to decline (T5, P4) (Figure 6A,B). In addition, FaesAP3_2a expression was similar with the FaesAP3_2 during the thrum and pin floral bud development (Figure 6A-C). Furthermore, FaesAP3_2 and FaesAP3_2a expression persisted in a relative high level during stamen filament elongation and anther development in pin and thrum flowers (T2-T4, P2-P3). 


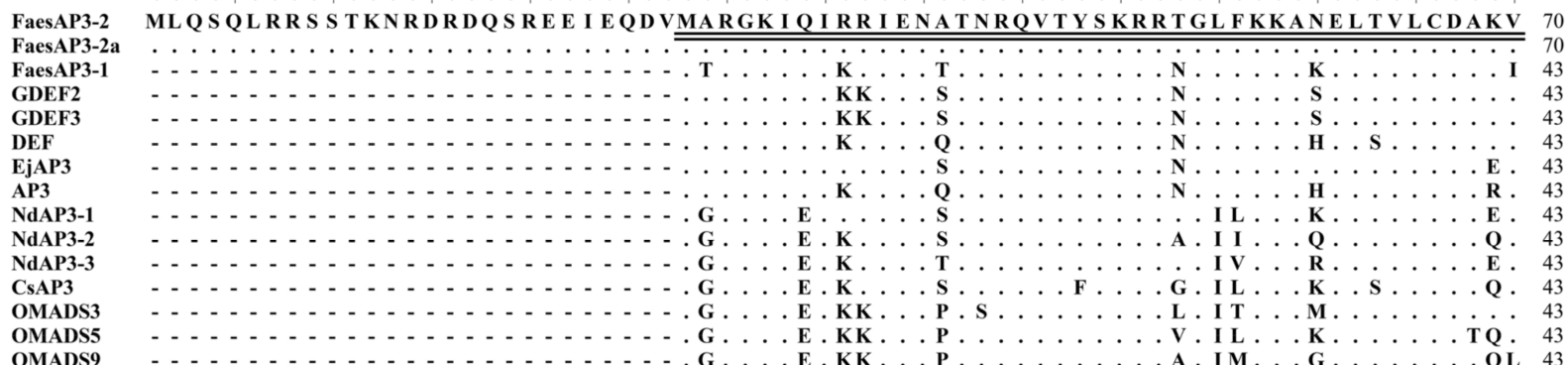

FaesAP3-2 FaesAP3-2 FaesAP3-1 GDEF2

GDEF3

DEF

EjAP3

AP3

NdAP3-2

NdAP3-3

CsAP3

CMADS 3

OMADS5

OMADS9

S I IMF SNTSKLHEF I S PNTT TKQMYDAYQNALGTD IWT S HYAKMQEDLEKLREANRQLQKE IRRRMGYCM 140

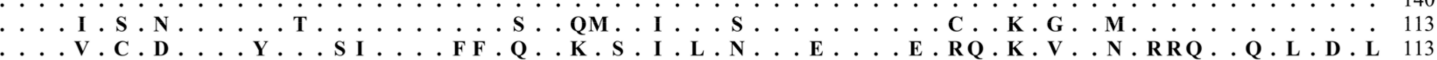

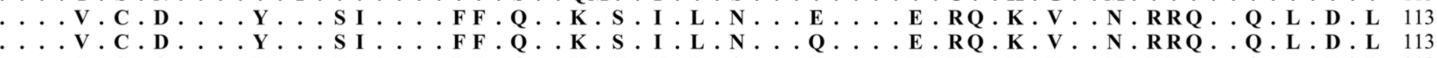

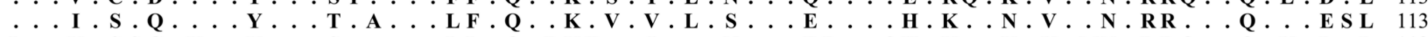

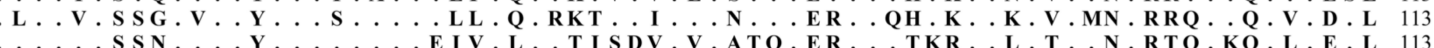

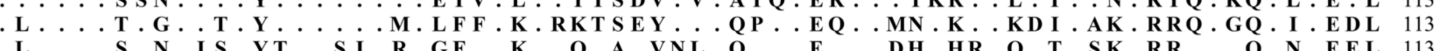

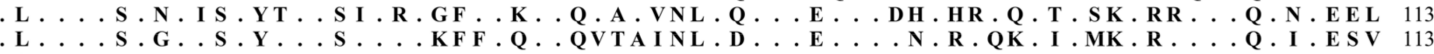

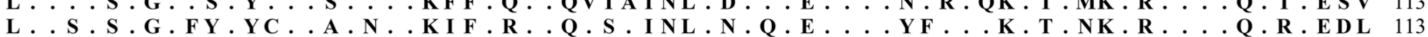

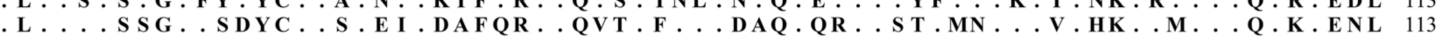

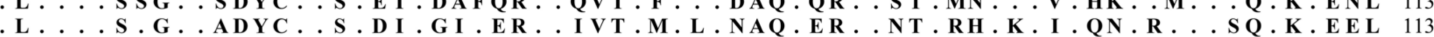

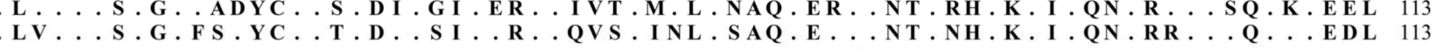
defgabcdefgabcdefgabcd

K 1

$\begin{array}{rrrrrr}150 & 160 & 170 & 180 & 190 & 200\end{array}$

FaesAP3-2 FaesAP3-2a FaesAP3-1 GDEF2 GDEF3 DEF

EjAP3

AP3

NdAP3-1

NdAP3-2

NdAP3-3

CsAP3

OMADS5

OMADS9

EDMNME ELVFLQHD I ENAVTN I RERKYKVLANQ I DT TKKKVRNGEG I HRSLMOAF - - - DVLREDPQYGLV 207 - - VSVAQHSF F - - 204

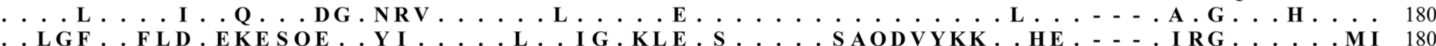

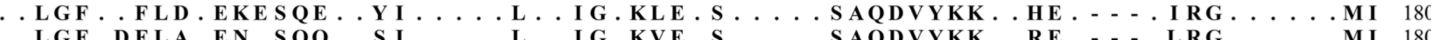

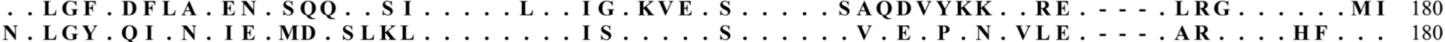

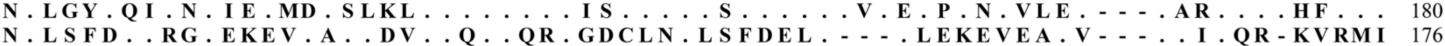

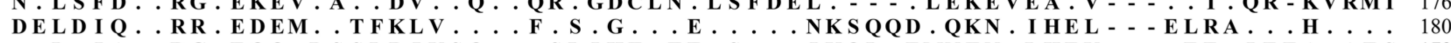

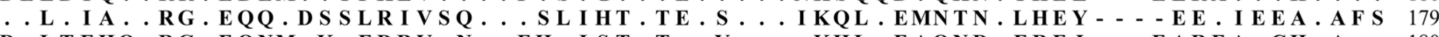

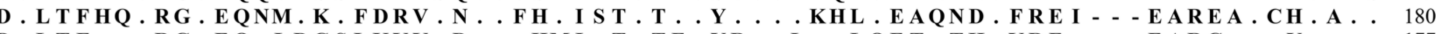

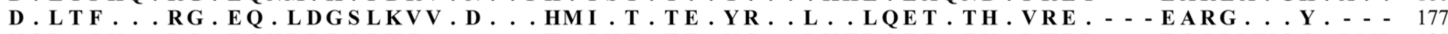

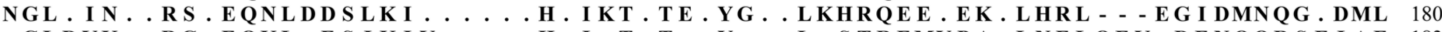

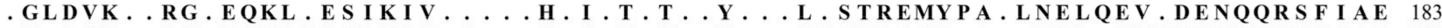

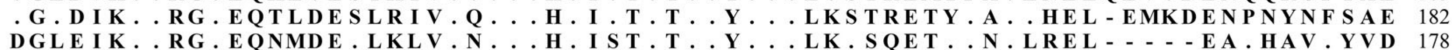
abcdefgabcdefga K2 defgabcdefgabcdefgabcdefgabcdefga K 3

220 240 250 $260 \quad$ euAP3 motif

\begin{tabular}{|c|c|c|c|c|}
\hline 3-2 & - KAGDY - - - NHV & LGL RW I PGD P N F P & SATA - - S CLTTYTYLE & \\
\hline$-\mathbf{2} \mathbf{a}$ & & & & 204 \\
\hline IesAP3-1 & DHML . L R . & H & & \\
\hline EF2 & DGVEYEALY. Y P PHIS A PRI &. $\mathrm{T}$ & & 228 \\
\hline DEF3 & DGRE Y E NMY . Y P - QMVA P R I & & H. A. GA & 231 \\
\hline & VL. F P - N NGGPR I & & H S C & 227 \\
\hline & KVK & & & 219 \\
\hline AP: & L . & & & 232 \\
\hline & H & ( & $\mathbf{I}$ & $210-20$ \\
\hline & I $\mathrm{T}$ & & L S & 225 \\
\hline & LL.MS - S GAG. L & & $\mathbf{L} \mathbf{R}$ & 221 \\
\hline & 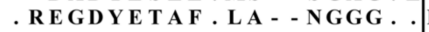 & F A & & 219 \\
\hline OM & . LS SGVYNSA I SMA - - NQ - - & $\ldots \mathrm{L}$ & & 204 \\
\hline & $-\mathrm{T}$ & & & 227 \\
\hline & 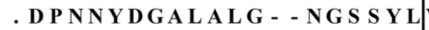 & 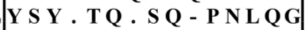 & S HDLR & 222 \\
\hline
\end{tabular}

OMADS5

\section{PI-derived motif paleoAP3 motif}

Figure 4. Sequence alignments of FaesAP3_2 and FaesAP3_2a with other AP3-like proteins from different clades of angiosperms. The double black underline represents the MADS domain and the single black underline the K domain. The conserved PI-derived motif, euAP3 motif and paleoAP3 motif located at the various C-terminal regions are boxed. The dots represent identical amino acid residues with FaesAP3_2. Dashes introduced into the sequence to improve the alignment. Three red underlines represent k1, k2 and k3 subdomains with (abcdefg)n repeats and usually with hydrophobic amino acids at positions a and $\mathrm{d}[19]$. 

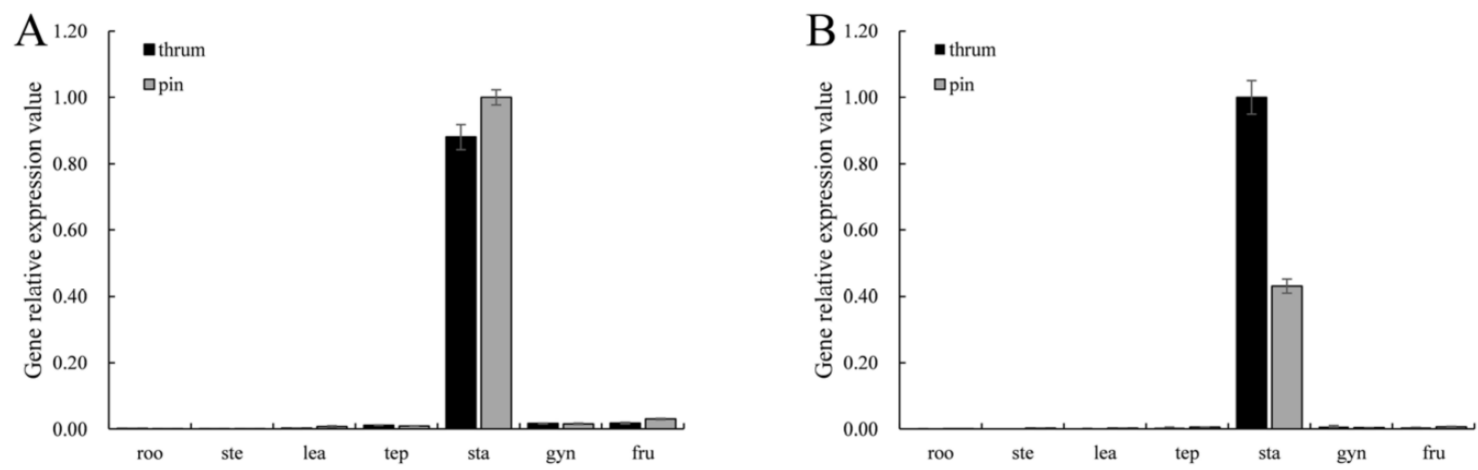

Figure 5. FaesAP3_2 and FaesAP3_2a expression in the roots (roo), stems (ste), juvenile leaves (lea), tepals (tep), stamens (sta), gynoecia (gyn) and 4-day-old fruits (fru) of F. esculentum by qRT-PCR with Faesactin as the internal control. (A) FaesAP3_2 expression in different tissues of pin and thrum flower plants were detected by qRT-PCR, respectively; (B) FaesAP3_2a expression in different tissues of pin and thrum flower plants were detected by qRT-PCR, respectively.

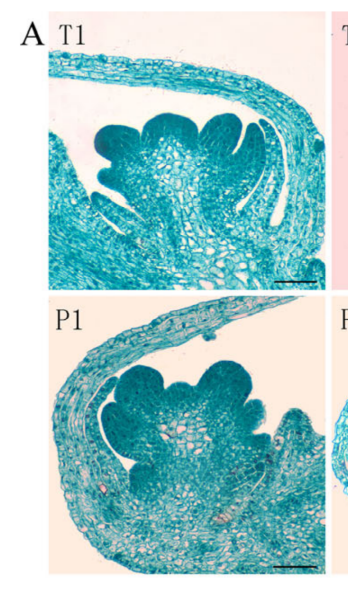

B

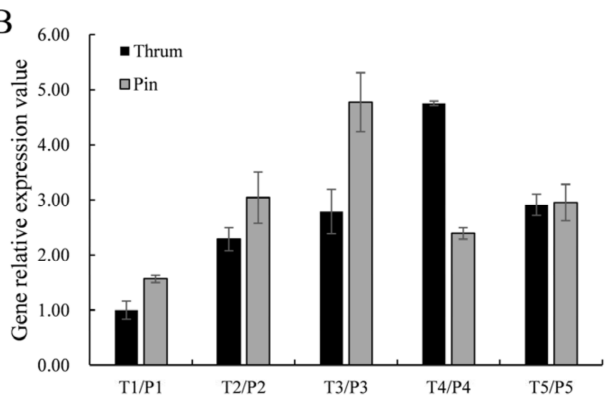

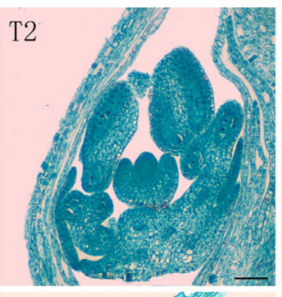
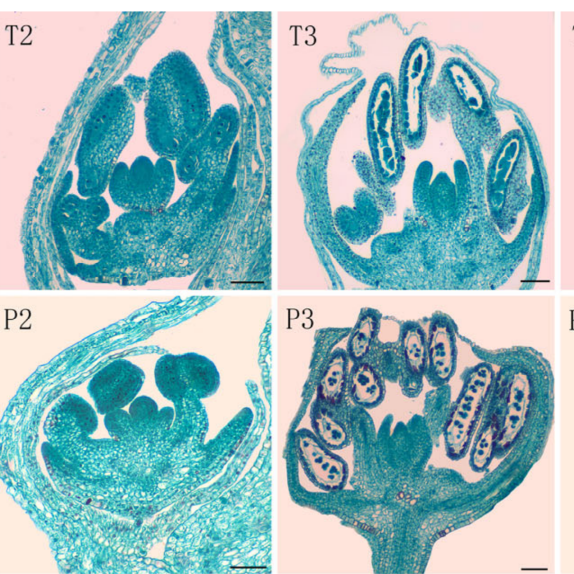

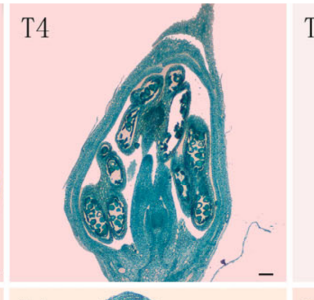

P4

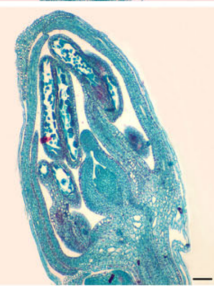

P5
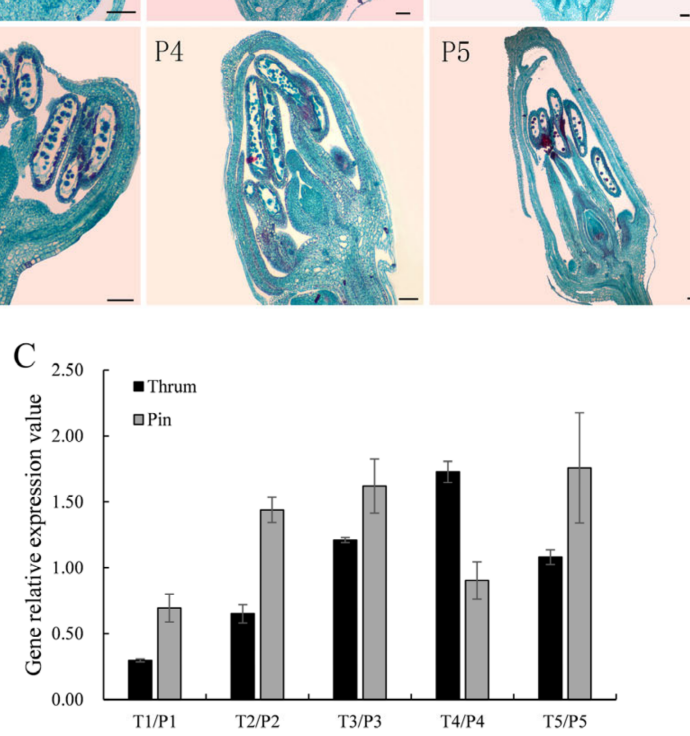

Figure 6. Morphology, FaesAP3_2 and FaesAP3_2a expression in common buckwheat distylous flowers at sequential developmental stages. (A) Morphology of thrum and pin flowers at various development stages; T1-T5: morphological differentiation and development of the thrum flower buds; T1: stamen primodium emergence; T2: stamen filament elongation; T3: microspore mother cells meiosis; T4: mononuclear microspore at Periphery, tepal enclosing; T5: full maturity flower buds with mature pollen and embryo sac before anthesis; P1-P5: morphological differentiation and development of the pin flower buds; P1: stamen primodium appearance; P2: stamen filament rapid elongating; P3: microspore mother cells meiosis and formation of microspores tetrads; P4: microspore released from tetrads and tepal enclosing; P5: full maturity flower bud with mature pollen and embryo sac before anthesis; (B) FaesAP3_2 expression at sequential development stages of thrum and pin flower buds were detected by qRT-PCR, respectively; (C) FaesAP3_2a expression at sequential development stages of thrum and pin flower buds were detected by qRT-PCR, respectively; scale bar: $100 \mu \mathrm{m}$.

\subsection{Isolation and Identification of FaesAP3_1 and FaesAP3_2 Promoters from F. esculentum}

A 2.4 kb pFaesAP3_1 (-2295/+135) (Genbank accession number: MK956946.1) and a 1.5 kb pFaesAP3_2 (-1401/+122) (Genbank accession number: MN016952.1) were iso- 
lated from F. esculentum, respectively. The putative transcription start site and cis-acting regulatory elements of both promoters were shown in Figures S1 and S2, respectively (Supplementary Figures S1-S2). Promoter pFaesAP3_1 contains a key CArG-box motif (-2160/-2151) for MADS-box transcription factor recognition and binding [20], which is also found in the promoter region of pFaesAP3_2 (-338/-329). In addition, each promoter contains two POLLEN2LELAT52 boxes and four GTGANTG10 boxes, which are essential for stamen-specific gene expression [21,22]. Furthermore, both promoters also contain binding sites (CCAATBOX1) for CONSTANS protein to regulate flowering [23]. Moreover, the MYCCONSENSUSAT box and ACGTATERD1 box are found in pFaesAP3_1 and pFaesAP3_2, which suggests that the corresponding gene expression could be induced by freezing/dehydration stress [24,25]. Some gibberellin-responsive elements, such as WRKY71OS-box, and PYRIMIDINEBOXOSRAMY1A/PYRIMIDINEBOXHVEPB box $[26,27]$, are also found in $p F a e s A P 3 \_1 / 2$, which suggested that the both genes could be regulated by gibberellin-responsive gene. In addition, several mesophyll-specific elements CACTFTPPCA1 boxes are also lying at pFaesAP3_1 and pFaesAP3_2 region, which suggested that the corresponding gene expression may extend to leaves [28].

A GUS reporter gene driven by pFaesAP3_1 or pFaesAP3_2 was activated in the cauline leaf, inflorescence rachis and flower of transgenic Arabidopsis (Figure 7B,C). Furthermore, GUS staining was observed in the flower where sepal, filament and stigma staining were intense, but was almost absent in petal, anther and stigmatic papillae of pFaesAP3_1::GUS transgenic Arabidopsis (Figure 7E). However, GUS staining was observed in the flower where sepal, stamen (filament and anther), stigma and stigmatic papillae staining were intense, but was almost absent in the petal of pFaesAP3_2::GUS transgenic Arabidopsis (Figure 7F).
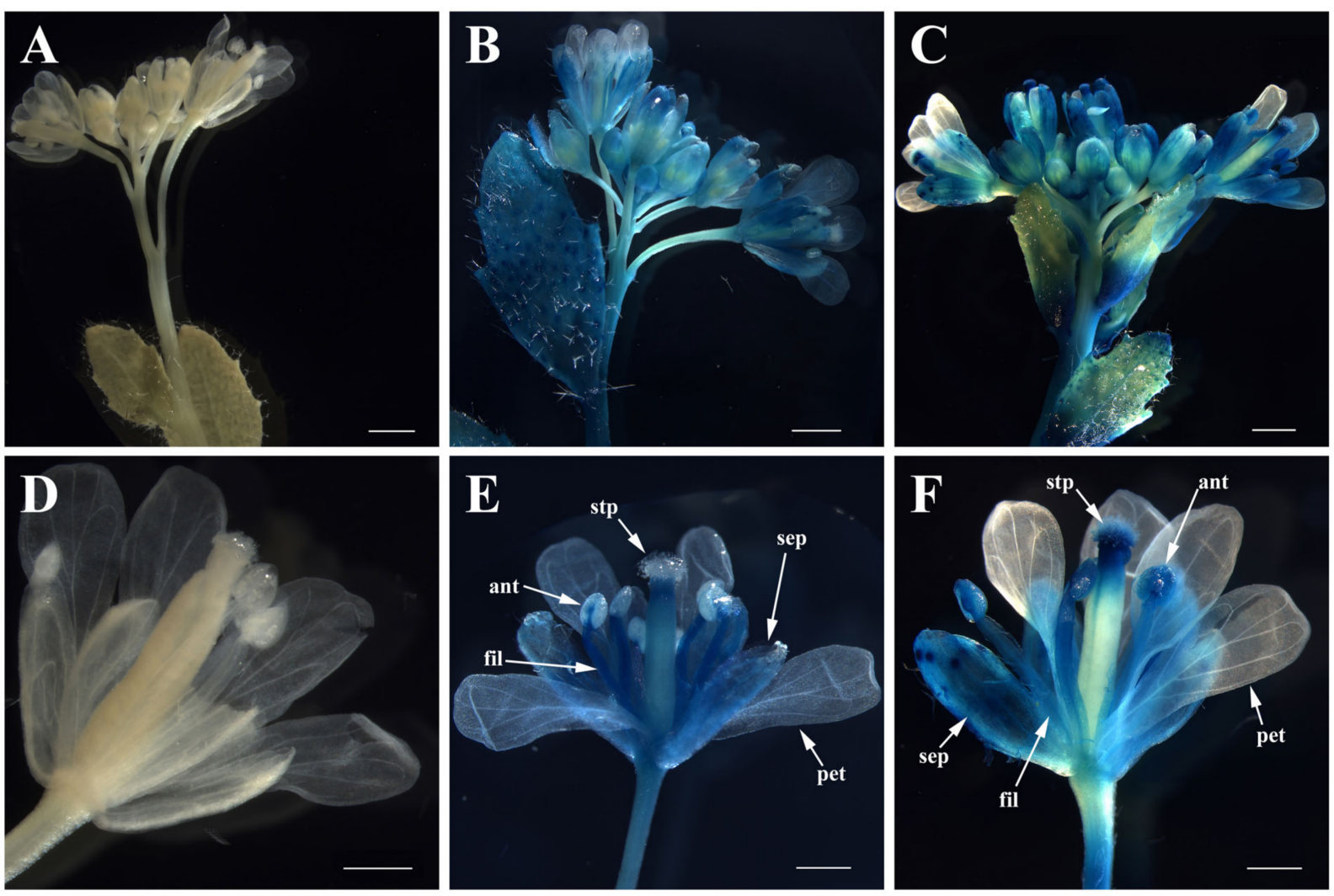

Figure 7. Histochemical GUS staining of pFaesAP3_1::GUS and pFaesAP3_2::GUS transgenic Arabidopsis, respectively. (A) Wild-type Arabidopsis inflorescence; (B) inflorescence of pFaesAP3_1::GUS transgenic Arabidopsis; (C) inflorescence of pFaesAP3_2::GUS transgenic Arabidopsis; (D) wild-type Arabidopsis flower; (E) flower of pFaesAP3_1::GUS transgenic Arabidopsis; (F) flower of pFaesAP3_2::GUS transgenic Arabidopsis. Sepal (sep), petal (pet), anther (ant), filament (fil), stigmatic papillae (stp); scale bars: (A-C) $1 \mathrm{~mm}$; (D-F) $500 \mu \mathrm{m}$. 


\subsection{Ectopic Expression of FaesAP3_2 and FaesAP3_2a in Arabidopsis ap3-3 Mutant}

To uncover the roles of FaesAP3_2 and FaesAP3_2a involved in floral development, 35S::FaesAP3_2 and 35S::FaesAP3_2a constructs have been transformed into heterozygote AP3/ap3-3 Arabidopsis to create complementation lines. All transgenic plants were confirmed by PCR and qRT-PCR. Furthermore, the independent transgenic lines of both 35S::FaesAP3_2 and 35S::FaesAP3_2a Arabidopsis under wild-type, heterozygote and homozygous background were confirmed by dCAPS method with $\mathrm{Cla}$ I restriction enzymes, respectively (Supplementary Figure S3). In addition, ectopic expression of FaesAP3_2 or FaesAP3_2a in transgenic lines under wild-type and homozygous background were detected, respectively. Moreover, 14 35S::FaesAP3_2 lines under wild-type background, 21 independent 35S::FaesAP3_2 lines under AP3/ap3-3 heterozygote background and 8 independent 35S::FaesAP3_2 lines under homozygous ap3 mutant background were obtained, respectively. In addition, $935 S$ ::FaesAP3_2a transgenic Arabidopsis plants under wildtype background, 16 35S::FaesAP3_2a transgenic Arabidopsis plants under heterozygote background and $1035 \mathrm{~S}::$ FaesAP3_2a transgenic Arabidopsis plants under homozygous background were also obtained, respectively. Phenotypes of different transgenic lines after flowering were assessed to evaluate whether FaesAP3_2 or FaesAP3_2a could replace the endogenous AP3 gene to control petal and stamen development in Arabidopsis ap3-3 mutant.

Among eight 35S::FaesAP3_2 transgenic ap3-3 Arabidopsis, two (25.00\%) showed a strong capability to rescue stamen-like organs in the third whorl of the flower (Figure 8E), one $(12.50 \%)$ displayed a medium capability to produce filament attached with carpelloid anther in whorl 3 (Figure $8 \mathrm{~F}$ ), two $(25.00 \%)$ with a weak complement phenotype have flowers with 1-2 filament-like organs in the stamen whorl (Figure 8G) and the remaining three lines (37.5\%) displayed no complementation and produced flowers similar to the flowers of Arabidopsis ap3-3 mutant (Figure 8H). Among 10 35S::FaesAP3_2a transgenic Arabidopsis ap3-3 mutants, 3 (30.00\%) completed stamen-like organ development in the third whorl of the flower (Figure $8 \mathrm{I}), 2(20.00 \%)$ with a medium-rescued phenotype produced flowers with filament attached with stigmatic papillae or carpelloid anther in whorl 3 (Figure $8 \mathrm{~J}$ ), two $(20.00 \%)$ displayed a weak capability to produce flowers only with filament-like organs or filament attached with stigmatic papillae in whorl 3 (Figure 8J) and the other three $(30.00 \%)$ lines showed no complementation phenotype (Figure $8 \mathrm{~K}$ ). In addition, neither 35S::FaesAP3_2 transgenic lines nor 35S::FaesAP3_2a transgenic lines under wild-type Arabidopsis background displayed flower phenotype change, and all the transgenic lines under wild-type Arabidopsis background produce flowers similar to the flowers of wild-type Arabidopsis (Figure 8B,C). Moreover, the expression levels of FaesAP3_2 and FaesAP3_2a corresponded to the degree of flower-phenotype complementation in transgenic ap3-3 Arabidopsis (Supplementary Figure S4). For example, FaesAP3_2 expression levels in transgenic line of homozygous ap3-3 Arabidopsis with strong complementation phenotype ( $\mathrm{T}_{1}-2-8 /$ ap3-3) were significantly higher than that of the weak complementation phenotype line $\left(\mathrm{T}_{1}-2-4 /\right.$ ap3-3) and no phenotype complementation line $\left(\mathrm{T}_{1}-2-2 / a p 3-3\right)$ $(L S D, p<0.05)$. Similar results were also observed in 35S::FaesAP3_2a transgenic ap3-3 Arabidopsis. For example, FaesAP3_2a expression levels in homozygous ap3-3 transgenic Arabidopsis with strong complementation phenotype ( $\mathrm{T}_{1}-2 \mathrm{a}-10 /$ ap3-3) were significantly higher than that of the weak complementation phenotype line $\left(\mathrm{T}_{1}-2 \mathrm{a}-5 / \mathrm{ap} 3-3\right)$ and no phenotype complementation line $\left(\mathrm{T}_{1}-2 \mathrm{a}-1 / \mathrm{ap} 3-3\right)(L S D, p<0.05)$. 

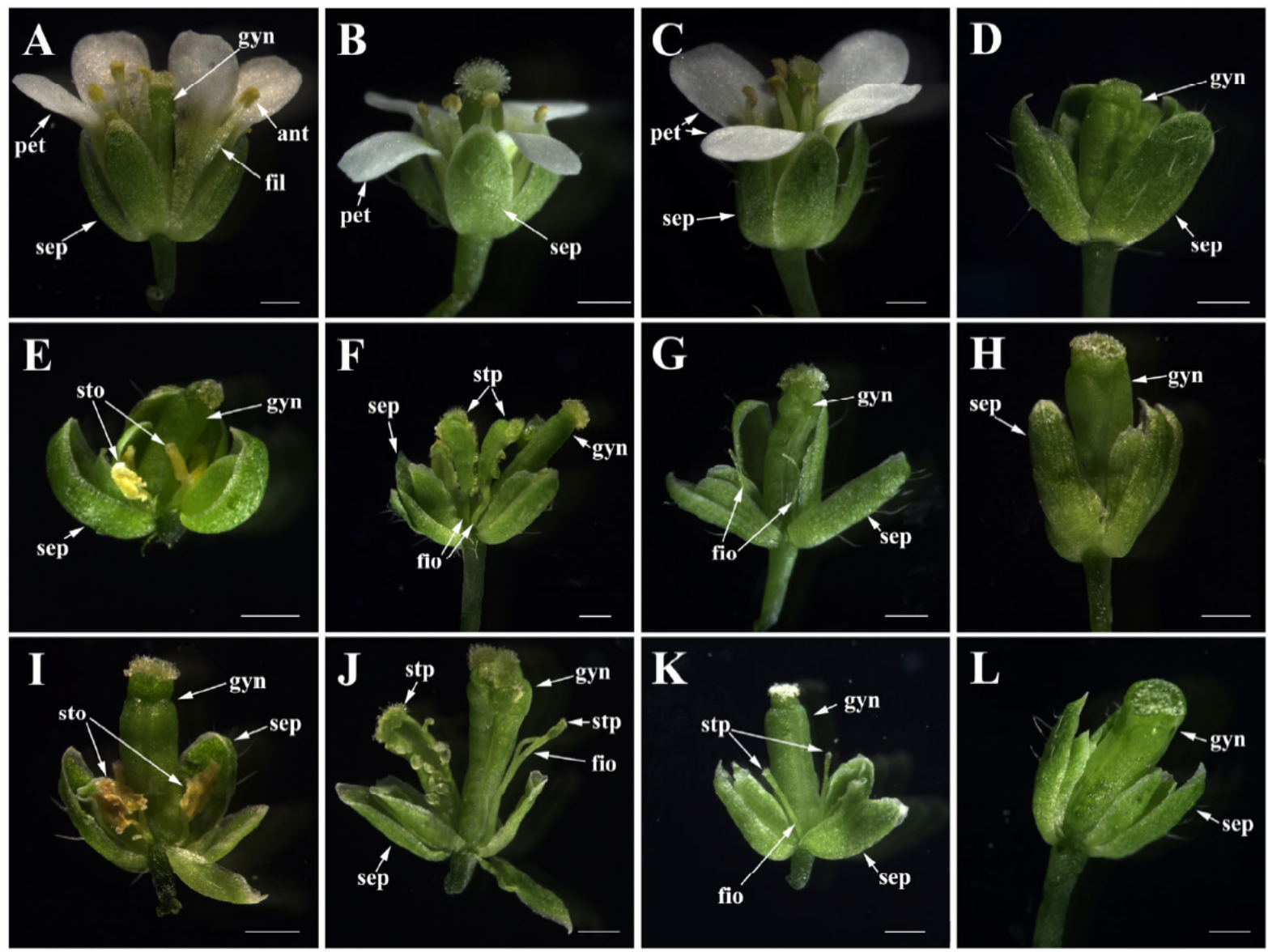

Figure 8. Flower phenotypes comparison of the wild-type, Arabidopsis ap3-3 mutant, 35S::FaesAP3_2 transgenic Arabidopsis ap3-3 mutant and 35S::FaesAP3_2a transgenic Arabidopsis ap3-3 mutant. (A) Wild-type Arabidopsis flower with normal flowers (sepal in whorl 1, petal in whorl 2, stamen in whorl 3 and fused carpel in whorl 4); (B) flower of 35S::FaesAP3_2 transgenic Arabidopsis under wild-type background; (C) flower of 35S::FaesAP3_2a transgenic Arabidopsis under wild-type background; (D) flower of Arabidopsis ap3-3 mutant with petal converted into sepal and stamen deficiency in whorl 3; (E) flower of 35S::FaesAP3_2 transgenic homozygous ap3-3 Arabidopsis with stamen-like organs in whorl 3; (F) flower of 35S::FaesAP3_2 transgenic homozygous ap3-3 Arabidopsis with filament attached with a carpelloid anther in whorl 3; (G) flower of 35S::FaesAP3_2 transgenic homozygous ap3-3 Arabidopsis with 1 2 filament-like organs in the stamen whorl; (H) flower of 35S::FaesAP3_2a transgenic homozygous ap3-3 Arabidopsis without phenotype complementation; (I) flower of 35S::FaesAP3_2a transgenic homozygous ap3-3 Arabidopsis with stamen-like organs in whorl 3; (J) flower of 35S::FaesAP3_2a transgenic homozygous ap3-3 Arabidopsis with filament attached with stigmatic papillae or carpelloid anther in whorl 3; (K) flower of 35S::FaesAP3_2a transgenic homozygous ap3-3 Arabidopsis only with filament-like organs or filament attached with stigmatic papillae in whorl 3; (L) flower of 35S::FaesAP3_2a transgenic homozygous ap3-3 Arabidopsis without phenotype complementation. Sepal (sep), petal (pet), anther (ant), filament (fil), filament-like organ (fio), stamen-like organ (sto), gynoecia (gyn), stigmatic papillae (stp); scale bars: $500 \mu \mathrm{m}$.

\section{Discussion}

Previous studies suggested that two paralogous lineages (euAP3 and TM6) of AP3-like genes in the core eudicots resulted from a duplication event of the ancestral paleoAP3 lineage within the basal eudicots [12]. Following the duplication, the euAP3 lineage acquired a novel C-terminal euAP3 motif instead of paleoAP3 motif and a new role in regulating petal development, while TM6 has preserved the C-terminal paleoAP3 motif [12,29]. In core eudicots, most euAP3-type genes, such as EjAP3 from Eriobotrya japonica [30], MtNMH7 from Medicago truncatula [31], PFDEF from Physalis floridana [32] and GDEF2 from Gerbera hybrid [33], were expressed only in petal and stamen, and were mainly involved in specifying petal and stamen identities during flower development. All these studies demonstrated that the functions of euAP3-type genes are highly correlated 
with their expression pattern in core eudicots. However, most TM6-like genes usually showed broader expression zones, but were involved only in stamen development in core eudicots. For examples, Medicago truncatula TM6-like gene MtTM6 was expressed predominantly in the outer cell layers of petal and stamen, but played a key role involving in stamen development [31]. Physalis floridana TM6-like gene PFTM6 was expressed in corolla, androecium and gynoecium, but was involved in pollen maturation [32]. Gerbera hybrid TM6-like GDEF1 was expressed in all four floral whorls of disk flower, but had a redundant role in determining stamen development [14]. Besides the broader expression patterns, small-scale gene duplication event of the paleoAP3-like genes were observed throughout basal eudicots with petaloid sepals and basal angiosperms with undifferentiated perianth (petaloid tepals). For examples, NdAP3-1, NdAP3-2 and NdAP3-3 were three paleoAP3type genes found in basal eudicots Nigella damascene (Ranunculaceae). NdAP3-3 was mainly expressed in petal and specified petal identity, while NdAP3-1 and NdAP3-2 have much broader expression domains (sepal, petal, stamen and carpel) and have overlapping functions in specifying stamen identity [33]. Three paleoAP3-type genes, AqAP3-1, AqAP3-2 and AqAP3-3, were also found in Aquilegia coerulea (Ranunculaceae). AqAP3-1 and AqAP3-2 were obviously expressed in sepals, petals, staminodia, stamens and carpels, and work together to specify stamen identity, while AqAP3-3 was mainly expressed in petals and was required only for petal identity [16]. Two paleoAP3-type genes, MAwuAP3_1 and MAwuAP3_2, were also found in basal angiosperms Magnolia wufengensis (magnoliids); both genes were mainly expressed in petaloid tepal and stamen, but were required only for stamen development [34]. All these data suggested that stamen-specific function of AP3-like genes antedate their petal-specific identity during angiosperm evolution.

Gene duplication events and AS are often associated with shifts in expression patterns and/or changes in coding sequence, giving rise to the diversification of gene function $[12,16,35]$. In F. esculentum, FaesAP3_1 was expressed only in stamen and exclusively required for stamen formation [9], while FaesAP3_2 expression extended to petaloid tepal and gynoecia although the expression was so slow. In addition, GUS staining was observed in the whole stamen of $p F a e s$ AP3_2::GUS transgenic Arabidopsis, while GUS staining was observed only in the filament of $p$ FaesAP3_1::GUS transgenic Arabidopsis, but was absent in the anther. All these data may suggest that FaesAP3_1 and FaesAP3_2 had overlapping functions in specifying stamen filament identity, and FaesAP3_2 played a key role in regulating anther development. Both genes work together to control normal stamen development. Intense GUS staining was also observed in the stigma and stigmatic papillae of $p$ FaesAP3_2::GUS transgenic Arabidopsis. Moreover, phenotype complementation analysis suggested that some 35S::FaesAP3_2 transgenic Arabidopsis ap3-3 mutants could produce flowers with a filament attached with stigmatic papillae in whorl 3 . These data may suggest that FaesAP3_2 may be involved in style development. However, the AS isoform FaesAP3_2a was expressed only in the stamen of thrum and pin flowers. Phenotype complementation analysis suggested that FaesAP3_2a holds a similar ability with FaesAP3_2 to rescue stamen development of Arabidopsis ap3-3 mutant even without the C-terminal euAP3 motif, which suggested that both AS isoforms had overlapping functions in specifying stamen identity of common buckwheat though they showed different expression patterns. As most transcriptional factors are modular proteins with multiple functional modules, some truncated protein isoforms containing functional modules may still have function and act as dominant-negative regulators [36]. The AP3-like and PI-like transcriptional factors are closely related MADS domain proteins that are thought to act as obligate heterodimers, and their I and $\mathrm{K}$ domains were required for dimerization and protein stability $[10,19]$. Hence, truncated FaesAP3_2a isoform might still participate in dimerization, which can compete with FaesAP3_2. A future challenge remains in exploring how the both AS isoforms work together with other common buckwheat $B$ genes to specify stamen identity. Moreover, previous studies also suggested that the petaloid tepal of common buckwheat were homologs to core eudicots sepal and showed a relative original trait of flowers [7,8]. All these data suggested that the MIK region of AP3-like proteins 
was crucial and essential for determining stamen identity, while the function of AP3-like proteins in specifying petal identity was gradually obtained accompanying sepal and petal differentiation after the AP3 orthologs acquired a novel C-terminal euAP3 motif during the evolution of core eudicots. Our data suggested early evolution of the functional specificity of euAP3-type proteins in floral organ development in core eudicots, and also provide an idea candidate gene for biotechnical engineering to develop a sterile male line of $F$. esculentum.

\section{Materials and Methods}

\subsection{Plant Material}

Thrum and pin floral buds at sequential developmental stages were sampled from common buckwheat 'Beizaosheng' growing under natural conditions in Jingzhou, China, respectively. In addition, each sample was divided into two aliquots; one was immediately frozen in liquid nitrogen, and then stored at $-80{ }^{\circ} \mathrm{C}$ until used; another was fixed in FAA (38\% formaldehyde: acetic acid: $70 \%$ ethanol $=1: 1: 18$, by volume). The root, stem, juvenile leaf, tepal, stamen, gynoecium and 4-day-old fruit of thrum and pin plants were dissected, respectively, immediately frozen in liquid nitrogen and then stored at $-80{ }^{\circ} \mathrm{C}$ until used. The Arabidopsis ap3-3 mutant line (CS3086, Landsberg ecotype) seeds were obtained from the ABRC (Arabidopsis Biological Resource Center, ABRC) at Ohio State University, Columbus, OH, USA.

\subsection{Isolation and Characterization of FaesAP3_2 and Its AS Isoform FaesAP3_2a from F. esculentum}

Total RNA from common buckwheat floral buds and the first-strand cDNA of $3^{\prime}$ RACE was prepared according to Fang et al. [9]. The $3^{\prime}$ end and $5^{\prime}$ partial cDNA sequences of two buckwheat FaesAP3_2 isoforms (FaesAP3_2 and FaesAP3_2a) were obtained by using the 3'-full RACE Core Set Ver. 2.0 kit (TaKaRa, Shiga, Japan) with gene-specific primer GSPAP3 based on the manufacturer's protocol (Supplementary Table S1). The gene-specific primer GSPAP3 was designed based on F01.PB7802 (putative AP3-like MADS-box transcription factor gene) of the BioProject ID PRJNA517031 deposited in the NCBI. Common buckwheat genomic DNA was extracted from leaves by using the CTAB Plant Genomic DNA Rapid Extraction Kit (Aidlab, Beijing, China) following the manufacturer's protocol. The full length of genomic DNA sequence of FaesAP3_2 was isolated from common buckwheat genomic DNA with the forward primer DFaesAP3_2F and the reverse primer DFaesAP3_2R. The PCR amplification of FaesAP3_2 genomic DNA was performed in a $25 \mu \mathrm{L}$ reaction volume containing $0.5 \mu \mathrm{L}$ Phanta Max Super Fidelity DNA Polymerase (Vazyme, Nanjing, China). PCR was performed with a 3 min $94{ }^{\circ} \mathrm{C}$ denaturation step, followed by 30 cycles of $30 \mathrm{~s}$ at $94^{\circ} \mathrm{C}, 30 \mathrm{~s}$ annealing at $58^{\circ} \mathrm{C}$, a $90 \mathrm{~s}$ extension at $72{ }^{\circ} \mathrm{C}$, with a final extension period of $10 \mathrm{~min}$. Sequence alignments and phylogenetic analysis of FaesAP3_2 were referenced to the method described by Liu et al. [37]. Putative FaesAP3_2 and FaesAP3_2a protein sequences, as well as B-class MADS-box transcription factors from different species, were selected for Phylogenetic trees from NCBI Genbank (Supplementary Table S2).

\subsection{Cytomorphological Examination and Expression Analysis of FaesAP3_2 and FaesAP3_2a}

The thrum and pin floral buds of F. esculentum fixed in FAA above were dehydrated in a graded ethanol series, cleared in a xylene series, infiltrated with molten paraffin, embedded into paraffin block, serially sectioned and then sections were stained according to Liu et al. [37]. The sections were observed under a CAIKON RCK-40C microscope and subsequently taken photomicrographs.

Total RNA of each sample was prepared for quantitative real-time PCR (qRT-PCR) by using the EASYspin plant RNA Rapid Extraction Kit (Aidlab, Beijing, China) following the manufacturer's protocol. The first-strand cDNA was synthesized by using the HiScript ${ }^{\circledR}$ II Q RT SuperMix for qPCR kit (Vazyme, Nanjing, China) according to the manufacturer's protocol. FaesAP3_2 and FaesAP3_2a expressions were detected in root, stem, juvenile leaf, 
tepal, stamen, gynoecium and 4-day-old fruit of thrum and pin plants by using qRT-PCR according to Liu et al. [8], but with the gene-specific forward primer qFaesAP3_2F and the gene-specific reverse primer qFaesAP3_2R for FaesAP3_2, and the gene-specific forward primer qFaesAP3_2aF and the gene-specific reverse primer qFaesAP3_2aR for FaesAP3_2a, respectively. Moreover, the expressions of FaesAP3_2 and FaesAP3_2a were also detected in different development stage floral buds of thrum and pin buckwheat through qRT-PCR suggested above, respectively. For qRT-PCR analysis, the reaction was performed on the Line-Gene 9600 Plus Real-time PCR Detection System by using $2 \times$ ChamQ SYBR qPCR Master Mix (Vazyme, Nanjing, China). Amplification fragment of F. esculentum actin gene (Genbank accession number: HQ398855.1) was used as the internal control with the forward primers qFaesactinF and the reverse qFaesactinR. qRT-PCR was carried out with three biological replicates, the PCR program was cycled and relative expression levels were calculated according to Liu et al. [8] but with $30 \mathrm{~s}$ annealing at $57^{\circ} \mathrm{C}$.

\subsection{Isolation and Identification of FaesAP3_1 and FaesAP3_2 Promoter from F. esculentum}

The FaesAP3_1 5' flanking region was isolated according to Liu et al. [8], but with the gene-specific primer D1pAP3_1SP1, D1pAP3_1SP2 and D1pAP3_1SP3 for the first walking sequencing, and with the gene-specific primer D2pAP3_1SP1, D2pAP3_1SP2 and D2pAP3_1SP3 for the second walking sequencing. In addition, The FaesAP3_2 5' flanking region was isolated according to the method suggested above but with the gene-specific primer FLpAP3_2SP1, FLpAP3_2SP2 and FLpAP3_2SP2 for the walking sequences. Moreover, the putative transcription start site of FaesAP3_1 was found based on the 5'RACE according to Fang et al. [9]. The putative transcription start site of FaesAP3_2 was found based on the $5^{\prime}$ RACE using the $5^{\prime}$ RACE System for Rapid Amplification of cDNA Ends (Invitrogen, Carlsbad, CA, USA) following the manufacturer's protocol and the genespecific primer 5RAP3GSP1, 5RAP3GSP2 and 5RAP3GSP3. The cis-acting elements lying at the $p$ FaesAP3_1 and $p$ FaesAP3_2 regions were searched in the PLACE database, respectively [38].

The $1.5 \mathrm{~kb} 5$ flanking region upstream of FaesAP3_2 translation start was cloned into pCAMBIA1300 vector with the forward primer TpFaesAP3_2F and the reverse primer TpFaesAP3_2R, and restriction enzymes Xba I and Sac I. pFaesAP3_2::GUS construct was transformed into A. thaliana Col-0 plants (ecotype Columbia) using the floral-dip method described by Clough and Bent [39]. Transgenic Arabidopsis seedlings were selected, cultivated and prepared for histochemical GUS staining according to Liu et al. [8].

\subsection{Ectopic Expression Analysis of FaesAP3_2 and FaesAP3_2a in Arabidopsis ap3-3 Mutant}

Full-length FaesAP3_2 and FaesAP3_2a cDNAs in the sense orientation were separately cloned into pBI121 vector with $\mathrm{Xba}$ I and $\mathrm{Sac}$ I restriction enzymes, and the forward primer TFaesAP3_2/2aF and the reverse primer TFaesAP3_2/2aR under control of the CaMV35S promoter using the ClonExpress ${ }^{\circledR}$ Ultra One Step Cloning Kit (Vazyme, Nanjing, China) according to the manufacturer's protocol. The 35S::FaesAP3_2 and 35S::FaesAP3_2a constructs were transformed into heterozygote Ap3/ap3-3 Arabidopsis using the floral-dip method described by Clough and Bent, respectively [39]. Transgenic Arabidopsis seeds were selected, and seedlings were cultivated according to Fang et al. [9]. Homozygous ap3-3 transgenic Arabidopsis lines were identified by dCAPS genotyping following the method suggested by Lamb and Irish [29]. The phenotypes of transgenic Arabidopsis were analyzed after flowering. In addition, the complementation degrees of independent transgenic lines of 35S::FaesAP3_2 and 35S::FaesAP3_2a homozygous ap3-3 Arabidopsis were categorized as 'no complementation', 'weak complementation', 'medium complementation' and 'strong complementation', respectively. Moreover, independent transgenic lines of each complementation degree were confirmed by qRT-PCR with the primers qTFaesAP3_2F and qTFaesAP3_2R for FaesAP3_2, and with the primers qFaesAP3_2a and qFaesAP3_2aR suggested above for FaesAP3_2a, respectively. Amplification fragment of A. thaliana ubiqui- 
tin 5 (Genbank accession number: NM_116090.3) with the primers qUBQ5F and qUBQ5R as the internal control.

\subsection{Statistical Treatment}

All experiments were carried out with three biological replicates, and data were expressed as mean $\pm \mathrm{SE}$ (standard errors). Statistical significance was determined by oneway ANOVA followed by $L S D$, and statistical significance was declared at $p$-value $\leq 0.01$ or 0.05 level, respectively.

\section{Conclusions}

Combined with our previous studies, we found that small-scale gene duplication (GD) event and alternative splicing (AS) of buckwheat AP3 orthologs resulted in FaesAP3_1, FaesAP3_2 and FaesAP3_2a. FaesAP3_2 and FaesAP3_2a were mainly expressed in the stamen of thrum and pin flowers. Promoters functional analysis suggested that intense GUS staining was observed in the whole stamen of $p F a e s A P 3 \_2:: G U S$ transgenic Arabidopsis, while intense GUS staining was observed only in the filament of $p F a e s A P 3$ _1::GUS transgenic Arabidopsis. These suggested that FaesAP3_1 and FaesAP3_2 had overlapping functions in specifying stamen filament identity and FaesAP3_2 played a key role in regulating anther development. Both genes work together to determine normal stamen development. Additionally, FaesAP3_2 and FaesAP3_2a owned the similar ability to rescue stamen development in Arabidopsis ap3-3 mutant, although AS resulted in frameshift mutation and consequent omission of the complete PI-derived motif and euAP3 motif of FaesAP3_2a. In addition, previous studies also suggested that the petaloid tepal of common buckwheat were homologs to core eudicots sepal. All these suggested that the MIK region of AP3-like proteins was crucial for determining stamen identity, while the function of AP3-like proteins in specifying petal identity was gradually obtained after the AP3 orthologs acquired a novel C-terminal euAP3 motif during the evolution of core eudicots. Our results also provide a clue to understanding the early evolution of the functional specificity of euAP3-type proteins in floral organ development in core eudicots, and also suggest that FaesAP3_2 holds the potential application for biotechnical engineering to develop a sterile male line of F. esculentum.

Supplementary Materials: The following are available online at https://www.mdpi.com/article/10 $.3390 /$ plants10081644/s1, Table S1: Primers used in this study. Table S2: Information on Sequences selected for alignments and phylogenetic analyses from NCBI GenBank. Figure S1: FaesAP3_1 promoter sequence. Figure S2: FaesAP3_2 promoter sequence. Figure S3: Genotyping of wildtype, heterozygous and homozygous ap3-3 mutant $A$. thaliana by dCAPS. Figure S4: Expression of FaesAP3_2 and FaesAP3_2a in transgenic Arabidopsis ap3-3 mutant confirmed by qRT-PCR.

Author Contributions: L.Z., writing—original draft preparation; L.Z., J.Z. and X.W., methodology; Z.L., writing-review and edition, supervision. All authors have read and agreed to the published version of the manuscript.

Funding: This work was supported by grant from the National Natural Science Foundation of China (Grant No. 31771867).

Institutional Review Board Statement: Not applicable.

Informed Consent Statement: Not applicable.

Data Availability Statement: All data generated or analyzed during this study are included in this published article. Further inquiries can be addressed to the corresponding author.

Acknowledgments: We thank Yangtze University. This work was supported by grant from the National Natural Science Foundation of China (Grant No. 31771867).

Conflicts of Interest: The authors declare no conflict of interest. 


\section{References}

1. Huda, N.; Lu, S.; Jahan, T.; Ding, M.; Jha, R.; Zhang, K.; Zhang, W.; Georgiev, M.I.; Park, S.U.; Zhou, M. Treasure from garden: Bioactive compounds of buckwheat. Food Chem. 2020, 335, 127653. [CrossRef]

2. Joshi, D.C.; Chaudhari, G.V.; Sood, S.; Kant, L.; Pattanayak, A.; Zhang, K.; Fan, Y.; Janovská, D.; Meglič, V.; Zhou, M. Revisiting the versatile buckwheat: Reinvigorating genetic gains through integrated breeding and genomics approach. Planta 2019, 250, 783-801. [CrossRef]

3. Li, L.-Y.; Fang, Z.-W.; Li, X.-F.; Liu, Z.-X. Isolation and characterization of the C-class MADS-box gene from the distylous pseudo-cereal Fagopyrum esculentum. J. Plant Biol. 2017, 60, 189-198. [CrossRef]

4. Matsui, K.; Yasui, Y. Buckwheat heteromorphic self-incompatibility: Genetics, genomics and application to breeding. Breed. Sci. 2020, 70, 32-38. [CrossRef]

5. Yasui, Y.; Mori, M.; Aii, J.; Abe, T.; Matsumoto, D.; Sato, S.; Hayashi, Y.; Ohnishi, O.; Ota, T. S-LOCUS EARLY FLOWERING 3 Is Exclusively Present in the Genomes of Short-Styled Buckwheat Plants that Exhibit Heteromorphic Self-Incompatibility. PLoS ONE 2012, 7, e31264. [CrossRef] [PubMed]

6. Yasui, Y.; Hirakawa, H.; Ueno, M.; Matsui, K.; Katsube-Tanaka, T.; Yang, S.J.; Aii, J.; Sato, S.; Mori, M. Assembly of the draft genome of buckwheat and its applications in identifying agronomically useful genes. DNA Res. 2016, 23, 215-224. [CrossRef]

7. Brockington, S.F.; Rudall, P.; Frohlich, M.W.; Oppenheimer, D.G.; Soltis, P.S.; Soltis, D.E. 'Living stones' reveal alternative petal identity programs within the core eudicots. Plant J. 2011, 69, 193-203. [CrossRef]

8. Liu, Z.; Fei, Y.; Zhang, K.; Fang, Z. Ectopic Expression of a Fagopyrum esculentum APETALA1 Ortholog only Rescues Sepal Development in Arabidopsis ap1 Mutant. Int. J. Mol. Sci. 2019, 20, 2021. [CrossRef] [PubMed]

9. Fang, Z.-W.; Qi, R.; Li, X.-F.; Liu, Z.-X. Ectopic expression of FaesAP3, a Fagopyrum esculentum (Polygonaceae) AP3 orthologous gene rescues stamen development in an Arabidopsis ap3 mutant. Gene 2014, 550, 200-206. [CrossRef] [PubMed]

10. Wuest, S.; Ó’Maoiléidigh, D.; Rae, L.; Kwasniewska, K.; Raganelli, A.; Hanczaryk, K.; Lohan, A.; Loftus, B.; Graciet, E.; Wellmer, F. Molecular basis for the specification of floral organs by APETALA3 and PISTILLATA. Proc. Natl. Acad. Sci. USA 2012, 109, 13452-13457. [CrossRef]

11. Kim, S.; Yoo, M.-J.; Albert, V.A.; Farris, J.S.; Soltis, P.S.; Soltis, D.E. Phylogeny and diversification of B-function MADS-box genes in angiosperms: Evolutionary and functional implications of a 260-million-year-old duplication. Am. J. Bot. 2004, 91, $2102-2118$. [CrossRef]

12. Kramer, E.M.; Su, H.-J.; Wu, C.-C.; Hu, J.-M. A simplified explanation for the frameshift mutation that created a novel C-terminal motif in the APETALA3 gene lineage. BMC Evol. Biol. 2006, 6, 30. [CrossRef]

13. Geuten, K.; Irish, V. Hidden Variability of Floral Homeotic B Genes in Solanaceae Provides a Molecular Basis for the Evolution of Novel Functions. Plant Cell 2010, 22, 2562-2578. [CrossRef]

14. Broholm, S.K.; Pöllänen, E.; Ruokolainen, S.; Tähtiharju, S.; Kotilainen, M.; Albert, V.A.; Elomaa, P.; Teeri, T.H. Functional characterization of B class MADS-box transcription factors in Gerbera hybrida. J. Exp. Bot. 2009, 61, 75-85. [CrossRef]

15. Nakatsuka, T.; Saito, M.; Nishihara, M. Functional characterization of duplicated B-class MADS-box genes in Japanese gentian. Plant Cell Rep. 2016, 35, 895-904. [CrossRef] [PubMed]

16. Sharma, B.; Kramer, E. Sub- and neo-functionalization of APETALA 3 paralogs have contributed to the evolution of novel floral organ identity in Aquilegia (columbine, Ranunculaceae). New Phytol. 2012, 197, 949-957. [CrossRef] [PubMed]

17. Chaudhary, S.; Khokhar, W.; Jabre, I.; Reddy, A.S.N.; Byrne, L.J.; Wilson, C.; Syed, N.H. Alternative Splicing and Protein Diversity: Plants Versus Animals. Front. Plant Sci. 2019, 10, 708. [CrossRef]

18. Nilsen, T.W.; Graveley, B.R. Expansion of the eukaryotic proteome by alternative splicing. Nature 2010, 463, 457-463. [CrossRef]

19. Yang, Y.; Jack, T. Defining subdomains of the K domain important for protein-protein interactions of plant MADS proteins. Plant Mol. Biol. 2004, 55, 45-59. [CrossRef]

20. de Folter, S.; Angenent, G.C. Trans meets cis in MADS science. Trends Plant Sci. 2006, 11, 224-231. [CrossRef] [PubMed]

21. Filichkin, S.A.; Leonard, J.M.; Monteros, A.; Liu, P.-P.; Nonogaki, H. A Novel Endo- $\beta$-Mannanase Gene in Tomato LeMAN5 Is Associated with Anther and Pollen Development. Plant Physiol. 2004, 134, 1080-1087. [CrossRef] [PubMed]

22. Rogers, H.; Bate, N.; Combe, J.; Sullivan, J.; Sweetman, J.; Swan, C.; Lonsdale, D.; Twell, D. Functional analysis of cis-regulatory elements within the promoter of the tobacco late pollen gene g10. Plant Mol. Biol. 2001, 45, 577-585. [CrossRef]

23. Wenkel, S.; Turck, F.; Singer, K.; Gissot, L.; Le Gourrierec, J.; Samach, A.; Coupland, G. CONSTANS and the CCAAT Box Binding Complex Share a Functionally Important Domain and Interact to Regulate Flowering of Arabidopsis. Plant Cell 2006, 18, 2971-2984. [CrossRef] [PubMed]

24. Agarwal, M.; Hao, Y.; Kapoor, A.; Dong, C.-H.; Fujii, H.; Zheng, X.; Zhu, J.-K. A R2R3 Type MYB Transcription Factor Is Involved in the Cold Regulation of CBF Genes and in Acquired Freezing Tolerance. J. Biol. Chem. 2006, 281, 37636-37645. [CrossRef]

25. Simpson, S.D.; Nakashima, K.; Narusaka, Y.; Seki, M.; Shinozaki, K.; Yamaguchi-Shinozaki, K. Two different novelcis-acting elements oferd1, a clpA homologous Arabidopsis gene function in induction by dehydration stress and dark-induced senescence. Plant J. 2003, 33, 259-270. [CrossRef]

26. Zhang, Z.-L.; Xie, Z.; Zou, X.; Casaretto, J.; Ho, T.-H.D.; Shen, Q.J. A Rice WRKY Gene Encodes a Transcriptional Repressor of the Gibberellin Signaling Pathway in Aleurone Cells. Plant Physiol. 2004, 134, 1500-1513. [CrossRef] [PubMed]

27. Mena, M.; Cejudo, F.J.; Isabel-Lamoneda, I.; Carbonero, P. A Role for the DOF Transcription Factor BPBF in the Regulation of Gibberellin-Responsive Genes in Barley Aleurone. Plant Physiol. 2002, 130, 111-119. [CrossRef] 
28. Gowik, U.; Burscheidt, J.; Akyildiz, M.; Schlue, U.; Koczor, M.; Streubel, M.; Westhoff, P. Cis-Regulatory Elements for MesophyllSpecific Gene Expression in the C4 Plant Flaveria trinervia, the Promoter of the C4 Phosphoenolpyruvate Carboxylase Gene. Plant Cell 2004, 16, 1077-1090. [CrossRef]

29. Lamb, R.S.; Irish, V.F. Functional divergence within the APETALA3/PISTILLATA floral homeotic gene lineages. Proc. Natl. Acad. Sci. USA 2003, 100, 6558-6563. [CrossRef]

30. Jing, D.; Chen, W.; Shi, M.; Wang, D.; Xia, Y.; He, Q.; Dang, J.; Guo, Q.; Liang, G. Ectopic expression of an Eriobotrya japonica APETALA3 ortholog rescues the petal and stamen identities in Arabidopsis ap3-3 mutant. Biochem. Biophys. Res. Commun. 2019, 523, 33-38. [CrossRef] [PubMed]

31. Roque, E.; Serwatowska, J.; Rochina, M.C.; Wen, J.; Mysore, K.; Yenush, L.; Beltrán, J.P.; Cañas, L.A. Functional specialization of duplicated AP3-like genes in Medicago truncatula. Plant J. 2012, 73, 663-675. [CrossRef]

32. Zhang, S.; Zhang, J.-S.; Zhao, J.; He, C. Distinct subfunctionalization and neofunctionalization of the B-class MADS-box genes in Physalis floridana. Planta 2014, 241, 387-402. [CrossRef]

33. Wang, P.; Liao, H.; Zhang, W.; Yu, X.; Zhang, R.; Shan, H.; Duan, X.; Yao, X.; Kong, H. Flexibility in the structure of spiral flowers and its underlying mechanisms. Nat. Plants 2015, 2, 15188. [CrossRef] [PubMed]

34. Jing, D.; Liu, Z.; Zhang, B.; Ma, J.; Han, Y.; Chen, F. Two ancestral APETALA3 homologs from the basal angiosperm Magnolia wufengensis (Magnoliaceae) can affect flower development of Arabidopsis. Gene 2014, 537, 100-107. [CrossRef] [PubMed]

35. Iñiguez, L.P.; Hernández, G. The Evolutionary Relationship between Alternative Splicing and Gene Duplication. Front. Genet. 2017, 8, 14. [CrossRef]

36. Reddy, A.S.; Marquez, Y.; Kalyna, M.; Barta, A. Complexity of the Alternative Splicing Landscape in Plants. Plant Cell 2013, 25, 3657-3683. [CrossRef] [PubMed]

37. Liu, Z.-X.; Xiong, H.-Y.; Li, L.-Y.; Fei, Y.-J. Functional Conservation of an AGAMOUS Orthologous Gene Controlling Reproductive Organ Development in the Gymnosperm Species Taxus chinensis var. mairei. J. Plant Biol. 2018, 61, 50-59. [CrossRef]

38. Higo, K.; Ugawa, Y.; Iwamoto, M.; Korenaga, T. Plant cis-acting regulatory DNA elements (PLACE) database: 1999. Nucleic Acids Res. 1999, 27, 297-300. [CrossRef]

39. Clough, S.J.; Bent, A. Floral dip: A simplified method forAgrobacterium-mediated transformation ofArabidopsis thaliana. Plant J. 1998, 16, 735-743. [CrossRef] 